\title{
Fuzzy Clustering applied to a Demand Response Model in a Smart Grid Contingency Scenario
}

\author{
R.Pereira ${ }^{1}$, R. Melício ${ }^{1}$, V.M.F. Mendes ${ }^{1}$, J. \\ Figueiredo $^{1}$, J. Martins ${ }^{2}$ \\ ${ }^{1}$ Mechatronics, Universidade de Évora, Portugal \\ ${ }^{2}$ FCT,Univ. Nova de Lisboa, Mt.Caparica, Portugal \\ ruimelicio@uevora
}

\author{
R. Pereira, A. Fagundes, V.M.F. Mendes, \\ J.C. Quadrado \\ Department of Electrical Engineering and Automation \\ Instituto Superior de Engenharia de Lisboa \\ Lisbon, Portugal \\ rpereira@deea.isel.pt
}

\begin{abstract}
This paper focus on a demand response model analysis in a smart grid context considering a contingency scenario. A fuzzy clustering technique is applied on the developed demand response model and an analysis is performed for the contingency scenario. Model considerations and architecture are described. The demand response developed model aims to support consumers decisions regarding their consumption needs and possible economic benefits.
\end{abstract}

Keywords—demand response; fuzzy clustering; smart grid; contingency

\section{INTRODUCTION}

Demand response (DR) techniques are included on demand side management strategies which also encompasses energy efficiency and energy conservation. These strategies are required to conveniently shape the load consumption diagram [1]. In a smart grid context, a consumer active role is essential for grid management in order to efficiently assure electric energy generation and usage, allowing to adequate the profile of energy load diagram to generation. As an example, timescheduling or load shedding requirements is improved when consumer assumes an active role. DR intends to conveniently shape energy usage in some specified time periods which are in association with economic benefits, to adequate the balance between load and generation. The shaping is achieved by a modus operandi efficiently taking into account the need to apply time-scheduling or load shedding. The modus operandi is intentional not only at lowering expenditures, such as the ones resulting from the need to call utilities expensive peaking power plants in short-time or to avoid building new power plant in order to satisfy future forecasted augmented energy consumption needs; but also in case of inability to avoid new power plants building, then mainly discarding the ones with manifestly impact on the environment, for instance, due to anthropogenic greenhouse gases emission [2]. In this paper the consumer actions in grid management are assisted by a DR model developed using a fuzzy clustering model. The designed methodology provides to the consumer a proposed efficient tool which allows sustaining consumer decisions on load management taking into consideration their consumption priorities and consumption needs along one day, regarding the associated costs for energy and possible economic benefits.
The desirable massive consumer adherence to DR actions leads to a consumption pattern definition for the model implementation. This consumption pattern substantiates the fuzzy clustering techniques applied. Domestic consumers are the target of the paper and are divided accordingly to their consumption profiles into three groups. Each group has an equipment priority list of Controllable Loads (CL) to be accomplished, considering for the decision on load satisfaction the available generation capacity and the energy price. Also, consumer non-controllable loads are considered [3].

This paper focuses on DR actions and DR model behaviour when a contingency scenario is considered. With this scenario an available power decreasing situation is considered. Details and results analysis are presented. The rest of the paper is organized as follows: Section II presents demand response as a demand side management strategy and the demand response model. Section III presents the fuzzy subtractive clustering method applied to the contingency scenario. Section IV presents the contingency description and analysis. Finally, concluding remarks are given in Section V.

\section{DEMAND RESPONSE}

\section{A. Demand response as a demand side management strategy}

The oil shock effects of the 70s triggered new research concerning policies and measures targeting energy demand. Since then energy efficiency policies and development of DR are on course, with the purpose to influence consumer quantities or patterns of energy [4]. In the 80s, a DSM discussion and analyze arise with alternatives associated to benefits on load shape derived from techniques such as: shifting of loads to off peak hours, clipping of peaks, valley filling of utility's loads strategic conservation to reduce demand, for example [5]. DSM techniques became more demanding with smart metering in domestic buildings and services and bidirectional communication provided in smart grids. DR enables a conveniently adaption of the load to the generation; this adaptation is essential due to the intermittent characteristic of renewable energy sources integrated into the electric grid. DR programs are mostly conditioned by grids requirements and consumers are driven to adapt the consumption in order to take advantage of economic benefits. 